\title{
Development and Validation of Stability-Indicating Method for Estimation of Chlorthalidone in Bulk and Tablets with the Use of Experimental Design in Forced Degradation Experiments
}

\author{
Sandeep Sonawane, Sneha Jadhav, Priya Rahade, Santosh Chhajed, and Sanjay Kshirsagar \\ MET's Institute of Pharmacy, MET League of Colleges, Bhujbal Knowledge City, Adgaon, Nashik, Maharashtra State 422003, India \\ Correspondence should be addressed to Sandeep Sonawane; sandeep.pharmacist@gmail.com
}

Received 21 December 2015; Revised 5 February 2016; Accepted 6 March 2016

Academic Editor: Maurizio Sanguinetti

Copyright (C) 2016 Sandeep Sonawane et al. This is an open access article distributed under the Creative Commons Attribution License, which permits unrestricted use, distribution, and reproduction in any medium, provided the original work is properly cited.

\begin{abstract}
Chlorthalidone was subjected to various forced degradation conditions. Substantial degradation of chlorthalidone was obtained in acid, alkali, and oxidative conditions. Further full factorial experimental design was applied for acid and alkali forced degradation conditions, in which strength of acid/alkali, temperature, and time of heating were considered as independent variables (factors) and \% degradation was considered as dependent variable (response). Factors responsible for acid and alkali degradation were statistically evaluated using Yates analysis and Pareto chart. Furthermore, using surface response curve, optimized $10 \%$ degradation was obtained. All chromatographic separation was carried out on Phenomenex HyperClone C 18 column $(250 \times 4.6 \mathrm{~mm}, 5 \mu)$, using mobile phase comprising methanol : acetonitrile : phosphate buffer $(20 \mathrm{mM})$ (pH 3.0 adjusted with $o$-phosphoric acid): $30: 10: 60 \%$ $\mathrm{v} / \mathrm{v}$. The flow rate was kept constant at $1 \mathrm{~mL} / \mathrm{min}$ and eluent was detected at $241 \mathrm{~nm}$. In calibration curve experiments, linearity was found to be in the range of $2-12 \mu \mathrm{g} / \mathrm{mL}$. Validation experiments proved good accuracy and precision of the method. Also there was no interference of excipients and degradation products at the retention time of chlorthalidone, indicating specificity of the method.
\end{abstract}

\section{Introduction}

A Stability-indicating assay method can be defined as "Validated quantitative analytical method that can detect the change with time in the chemical, physical or microbiological properties of the drug substance and drug products are specific so that the content of active ingredients and degradation products can be accurately measured without interference" [1].

Generally forced degradation/stress testing is used to generate the samples for stability-indicating assay methods. Forced degradation/stress testing is defined as "the stability testing of drug substance and drug product under conditions exceeding those used for accelerated stability testing" [2]. Degradation can be achieved by exposing the drug, for extended period of time, to extremes of $\mathrm{pH}(\mathrm{HCl}$ or $\mathrm{NaOH}$ solutions of different strengths), at elevated temperature, to hydrogen peroxide at room temperature, to UV light, and to dry heat (in an oven) to achieve degradation to an extent of $5-20 \%$. Generally, trial and error experimentation is used during these experiments. This trial and error approach is generally cost, labor, and time intensive and should be substituted with some systematic approach. From exhaustive literature, it was observed that experimental design in forced degradation experiments can be used to save cost and labor by avoiding trial and error experimentation $[3,4]$.

General steps involved in experimental design strategy are the selection of variables, selection of response, selection of design, preparation of experimental domain, preparation of experimental matrix, generation of polynomial equation, screening the important/most affecting variables using either one-way ANOVA or Pareto chart or using normal/half normal plots, and finally selection of optimum region by surface response.

The aim of the present work was to implement experimental design strategy in forced degradation experimentation and to arrive at optimum degradation conditions. For the same, chlorthalidone was selected as a model drug. 
<smiles>NS(=O)(=O)c1cc(C2(O)NC(=O)c3ccccc32)ccc1Cl</smiles>

FIGURE 1: Chemical structure of chlorthalidone.

Full factorial experimental design was used during acid and alkali degradation experiments. After determining the polynomial equations, significant factors were determined using ANOVA and Pareto chart and finally the optimum regions for acid and alkali degradation conditions were determined using surface response curve.

Chemically, chlorthalidone is 2-chloro-5-(2,3-dihydro-1hydroxy-3-oxo-1H-isoindol-1-yl) benzenesulfonamide (Figure 1) [5]. It is an antihypertensive and diuretic drug used alone or in combination with other drugs to treat hypertension and various renal disorders. Literature survey revealed that few stability-indicating methods are reported for the estimation of chlorthalidone in combination with other drugs [6-8].

\section{Materials and Methods}

2.1. Chemicals and Reagents. Pharmaceutical grade chlorthalidone was supplied as a gift sample from Trichem Laboratories Ltd., Mumbai, India. Methanol and acetonitrile used in analysis were of HPLC grade and all other chemicals and reagents were of analytical grade and were purchased from SD Fine Chemicals, Mumbai, India. Double distilled water used was freshly prepared by Double Distillation Assembly (Borosil, Mumbai, India) and further used in analysis after filtering through $0.45 \mu$ membrane filter papers purchased from Millipore (India) Pvt. Ltd., Bengaluru, India. Chlorthalidone tablets (label claim $12.5 \mathrm{mg} /$ tablet) were purchased from local market.

2.2. Apparatus and Equipment. HPLC instrumentation consisting of pump PU-2080 plus (JASCO, Tokyo, Japan), with Rheodyne manual loop injector $7725 i$ (injection loop capacity $20 \mu \mathrm{L}$ ) was used. Detection was carried out using UV-2075 detector (JASCO, Tokyo, Japan). Data acquisition was done by Borwin chromatography software version 1.5 (JASCO, Tokyo, Japan). All calculations were performed using Microsoft Excel 2010 (Microsoft Corporation).

2.3. Chromatographic Conditions. All chromatographic separations were carried out on Phenomenex HyperClone C 18 column $(250 \times 4.6 \mathrm{~mm}, 5 \mu)$, using mobile phase comprising methanol: acetonitrile:phosphate buffer $(20 \mathrm{mM})(\mathrm{pH} 3.0$ adjusted with $o$-phosphoric acid): $30: 10: 60 \% \mathrm{v} / \mathrm{v}$. The flow rate was kept constant throughout analysis at $1 \mathrm{~mL} / \mathrm{min}$ and eluent was detected at $241 \mathrm{~nm}$.

Forced degradation experiments were carried out on chlorthalidone under various conditions explained in $\mathrm{ICH}$ guideline Q1A(R2), namely, acid, alkali, wet heat, dry heat, and oxidative and photolytic conditions [9]. Full factorial experimental design was used for acid and alkali forced degradation:

(a) Acid degradation: $1 \mathrm{mg} / \mathrm{mL}$ mixture of chlorthalidone in $X_{1} \mathrm{M} \mathrm{HCl}$ was heated under reflux at $X_{2}{ }^{\circ} \mathrm{C}$ for $X_{3}$ min. Three factors, strength of acid $\left(X_{1}\right)$, temperature of heating $\left(X_{2}\right)$, and time of exposure $\left(X_{3}\right)$, were studied at two levels. The high level $(+1)$ for $X_{1}, X_{2}$, and $X_{3}$ was $0.1 \mathrm{M}, 80^{\circ} \mathrm{C}$, and $60 \mathrm{~min}$, respectively, and the low level $(-1)$ for $X_{1}, X_{2}$, and $X_{3}$ was $0.01 \mathrm{M}$, $55^{\circ} \mathrm{C}$, and $30 \mathrm{~min}$, respectively. As three factors were studied at two levels, a $2^{3}$ factorial design was used and experimental matrix of eight experiments was prepared with combination of each factor at each level.

(b) Alkali degradation: $1 \mathrm{mg} / \mathrm{mL}$ mixture of chlorthalidone in $X_{1} \mathrm{M} \mathrm{NaOH}$ was heated under reflux at $X_{2}{ }^{\circ} \mathrm{C}$ for $X_{3}$ min. Three factors, strength of alkali $\left(X_{1}\right)$, temperature of heating $\left(X_{2}\right)$, and time of exposure $\left(X_{3}\right)$, were studied at two levels. The high level $(+1)$ for $X_{1}, X_{2}$, and $X_{3}$ was $0.1 \mathrm{M}, 80^{\circ} \mathrm{C}$, and $30 \mathrm{~min}$, respectively, and the low level $(-1)$ for $X_{1}, X_{2}$, and $X_{3}$ was $0.01 \mathrm{M}, 55^{\circ} \mathrm{C}$, and $15 \mathrm{~min}$, respectively. As three factors were studied at two levels, a $2^{3}$ factorial design was used and experimental matrix of eight experiments was prepared with combination of each factor.

(c) Wet heat degradation: $1 \mathrm{mg} / \mathrm{mL}$ mixture of chlorthalidone in water was heated under reflux at $80^{\circ} \mathrm{C}$ for $48 \mathrm{hr}$.

(d) Oxidative degradation: $1 \mathrm{mg} / \mathrm{mL}$ mixture of chlorthalidone in $30 \%$ of $\mathrm{H}_{2} \mathrm{O}_{2}$ was kept under dark at room temperature for $48 \mathrm{hr}$.

(e) Dry heat degradation: $10 \mathrm{mg}$ of chlorthalidone was spread as a thin film in Petri plate and placed in a hot air oven set at a temperature of $80^{\circ} \mathrm{C}$ for $24 \mathrm{hr}$.

(f) Photolytic degradation: chlorthalidone powder was spread as a thin layer in Petri plate and exposed to direct sunlight for 7 days.

2.4. Chromatographic Analysis of Forced Degraded Samples. After degradation, each sample obtained under each forced degradation condition was diluted appropriately with mobile phase to get a final concentration of $10 \mu \mathrm{g} / \mathrm{mL}$; the resulting solution was injected in the column under described chromatographic condition. The chromatogram obtained was studied for area of drug peak and appearance of secondary peaks. The decrease in the area of the drug peak and the occurrence of secondary peaks was considered as indication of degradation. The $\%$ degradation was calculated as

$\%$ degradation

$$
=\frac{\text { area of unstressed }- \text { area of stressed }}{\text { area of unstressed }} \times 100 .
$$


2.5. Calibration Curve. Standard stock solution of chlorthalidone was prepared in methanol to obtain a concentration of $1 \mathrm{mg} / \mathrm{mL}$. The resulting solutions were diluted with mobile phase to get concentrations in the range of $2-12 \mu \mathrm{g} / \mathrm{mL}$ and each solution was subjected to chromatographic analysis in triplicate under mentioned chromatographic conditions. The peak areas were plotted on $y$-axis and their respective concentrations were plotted on $x$-axis. Furthermore, the linear regression was performed to generate least square line and regression equation of type:

$$
y=a x+b .
$$

2.6. Method Validation. Analytical method validation was carried out as per ICH method validation guideline Q2(R1) [10]. The method was validated for specificity, accuracy, precision, limit of detection (DL), and limit of quantitation (QL). Accuracy and precision were evaluated by fortifying a placebo with amounts of drug corresponding to $80 \%, 100 \%$, and $120 \%$ of label claimed and analyzing the resulting mixture in triplicate over three days.

2.7. Analysis of Formulation. Twenty tablets were weighed and finely powdered. A quantity of powder equivalent to $10 \mathrm{mg}$ of chlorthalidone was transferred to $100 \mathrm{~mL}$ volumetric flask and was sonicated with $80 \mathrm{~mL}$ methanol for $10 \mathrm{~min}$. The excipients were separated by filtration and the volume was made up to the mark with the same solvent. From the resulting solution, $1 \mathrm{~mL}$ aliquot was removed and transferred to $10 \mathrm{~mL}$ volumetric flask. The volume was made up to the mark with mobile phase to get a $10 \mu \mathrm{g} / \mathrm{mL}$ solution. The resulting solution was subjected to chromatographic analysis in triplicate. The drug peak area was referred to linear regression equation to get the sample concentration and nominal $\%$ of label claim.

\section{Results and Discussion}

3.1. Optimization of Mobile Phase. To get adequate retention and resolution of chlorthalidone and its formed degradation products under various forced degradation conditions, mobile phases of different strengths and $\mathrm{pH}$ were tried. All buffer solutions used in mobile phases were prepared as per the procedure described in Synder et al. [11]. From the chromatograms obtained, it was concluded that the mobile phase consisting of methanol:acetonitrile: $20 \mathrm{mM}$ phosphate buffer ( $\mathrm{pH}$ 3.0) (30: 10: 60\% v/v) gave adequate retention of chlorthalidone and also resolved chlorthalidone from its degradation products formed under various forced degradation conditions. The maximum absorption wavelength of chlorthalidone and its degradation products was found to be $241 \mathrm{~nm}$.

3.2. Forced Degradation Behavior of Chlorthalidone. When forced degradation experiments were performed and subjected to chromatographic analysis, it was found that chlorthalidone was stable to wet heat, dry heat, and photolytic conditions but substantial degradation was obtained under acid, alkali, and oxidative conditions. Under acid degradation condition, two degradation products were found at $11.07 \mathrm{~min}$ and $13.9 \mathrm{~min}$ and under alkali degradation condition, one degradation product was obtained at $11.49 \mathrm{~min}$. When drug was subjected to oxidative degradation condition for $48 \mathrm{hrs}$, one degradation product was obtained at $6.55 \mathrm{~min}$. The chromatograms of unstressed chlorthalidone and chlorthalidone exposed to acid degradation, alkali degradation, and oxidative condition are shown in Figures 2(a), 2(b), 2(c), and $2(\mathrm{~d})$, respectively.

3.3. Experimental Design in Acid and Alkali Forced Degradation Conditions. From the experimental matrix constructed (Table 1) as per the experimental conditions discussed for acid and alkali degradation conditions and from the \% degradation obtained for each experiment, polynomial equations were obtained for acid and alkali degradation, respectively.

The general polynomial equation for $2^{3}$ factorial design is

$$
\begin{aligned}
Y= & \beta_{0}+\beta_{1} X_{1}+\beta_{2} X_{2}+\beta_{3} X_{3}+\beta_{12} X_{1} X_{2}+\beta_{23} X_{2} X_{3} \\
& +\beta_{13} X_{1} X_{3}+\beta_{123} X_{1} X_{2} X_{3},
\end{aligned}
$$

where $Y$ is the response (\% degradation), $\beta_{0}$ is intercept, and $\beta_{1}, \beta_{2}$, and $\beta_{3}$ are coefficients for variables $X_{1}, X_{2}$, and $X_{3}$, respectively. Similarly $\beta_{12}, \beta_{23}, \beta_{13}$, and $\beta_{123}$ are the coefficients for interaction of variables, $X_{1}$ and $X_{2}, X_{2}$ and $X_{3}, X_{1}$ and $X_{3}$, and $X_{1}, X_{2}$, and $X_{3}$, respectively.

To obtain the polynomial equations for acid and alkali degradation condition, the value of each coefficient and intercept for each degradation condition was determined.

The intercept $\left(\beta_{0}\right)$ for polynomial equation was calculated as

$$
\beta_{0}=\frac{\sum X Y}{2^{n}}
$$

and the respective coefficients were calculated as

$$
\beta_{1}=\frac{\sum X_{1} Y}{2^{n}}
$$

where $X_{1}$ is the value of the column and $Y$ is the response (\% degradation of the drug).

All other coefficients were obtained in a similar manner and the polynomial equations were obtained. The polynomial equations for acid and alkali degradation were obtained as follows.

The polynomial equation for acid degradation is

$$
\begin{aligned}
Y= & 18.32-0.67 X_{1}+10.26 X_{2}+4.56 X_{3}-0.16 X_{1} X_{2} \\
& +2.59 X_{2} X_{3}+0.44 X_{1} X_{3}-0.10 X_{1} X_{2} X_{3} .
\end{aligned}
$$

The polynomial equation for alkali degradation is

$$
\begin{aligned}
Y= & 19.64+0.87 X_{1}+10.88 X_{2}+3.08 X_{3}+0.67 X_{1} X_{2} \\
& +0.87 X_{2} X_{3}-0.25 X_{1} X_{3}+0.67 X_{1} X_{2} X_{3} .
\end{aligned}
$$

Furthermore, to obtain the regression of the polynomial equations (see (6) and (7)) and to determine the significant 


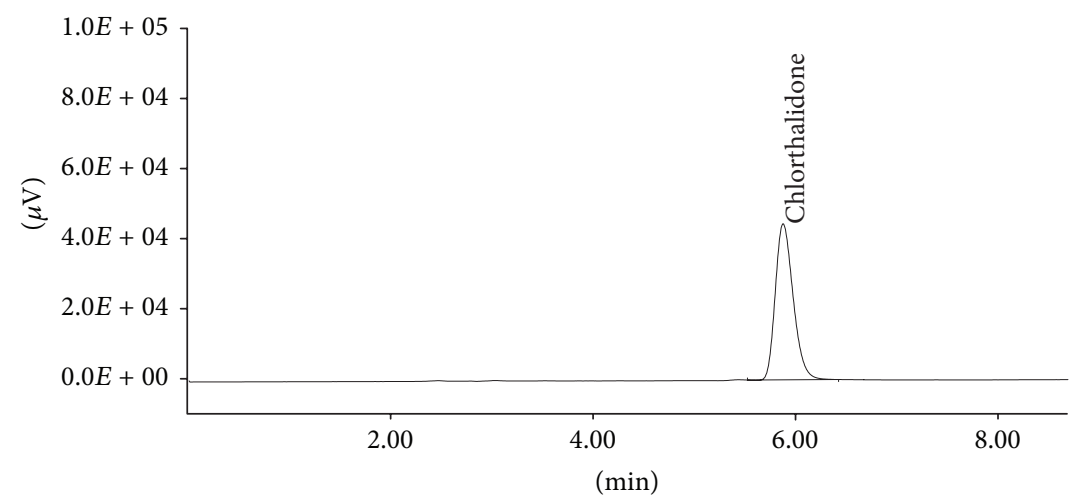

(a)

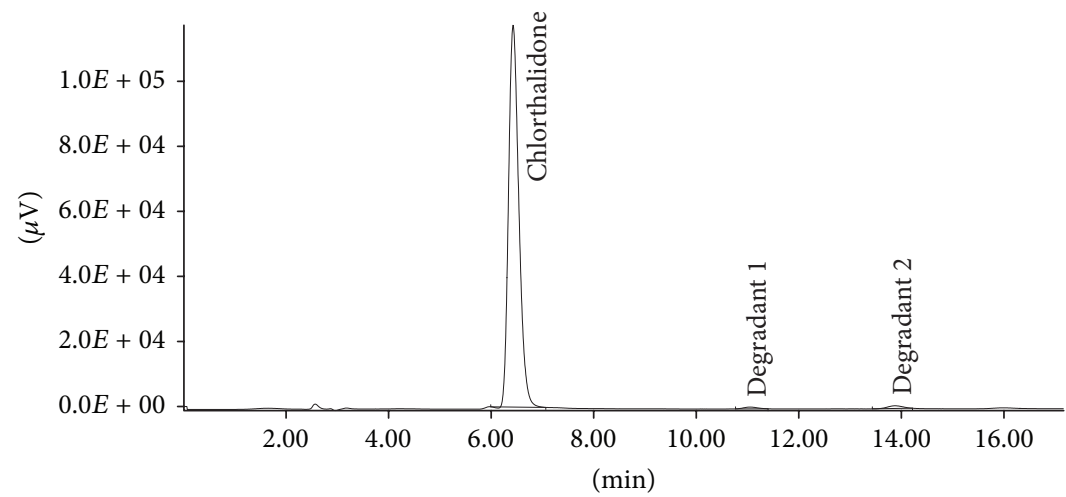

(b)

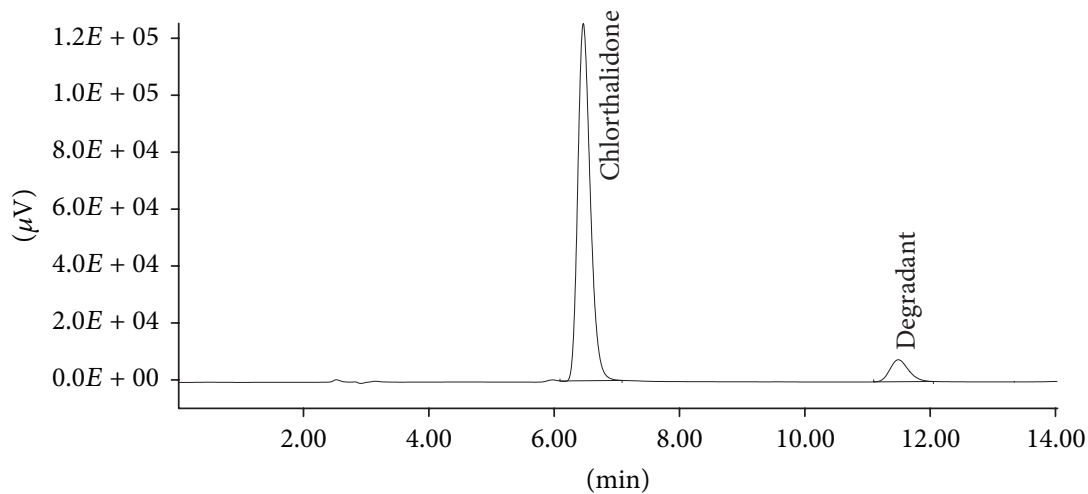

(c)

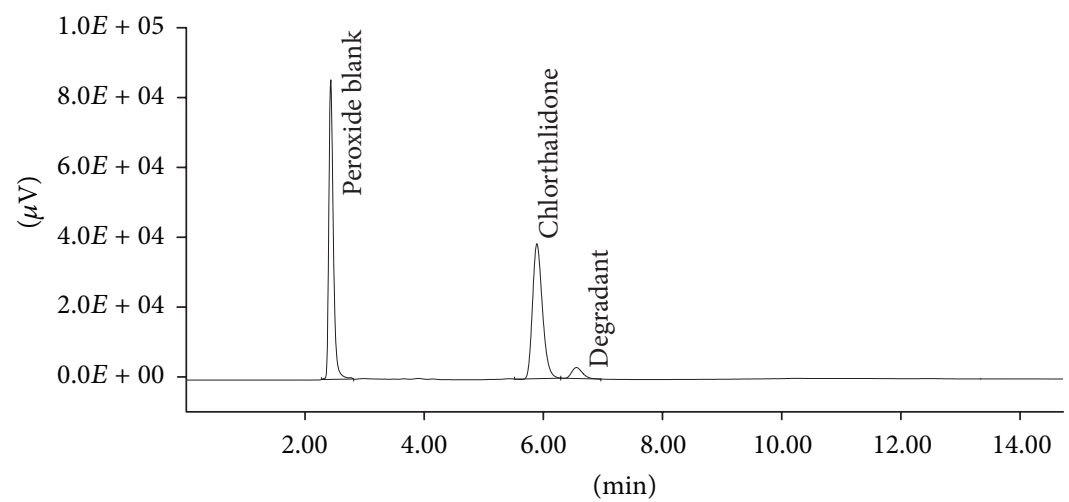

(d)

FIGURE 2: (a) Representative chromatogram of unstressed chlorthalidone. (b) Representative chromatogram of acid treated chlorthalidone. (c) Representative chromatogram of alkali treated chlorthalidone. (d) Representative chromatogram of oxidative degradation of chlorthalidone. 
TABLE 1: Experimental matrix of $2^{3}$ factorial design for acid and alkali degradation.

\begin{tabular}{|c|c|c|c|c|c|c|c|c|c|c|}
\hline \multirow{2}{*}{ Expt. number } & \multicolumn{3}{|c|}{ Levels of factor in experiment } & \multicolumn{4}{|c|}{ Interaction } & \multirow{2}{*}{ Total } & \multirow{2}{*}{$\begin{array}{c}\% \\
\text { acid deg. }\end{array}$} & \multirow{2}{*}{$\begin{array}{c}\% \\
\text { alkali deg } \\
\end{array}$} \\
\hline & $X_{1}$ & $X_{2}$ & $X_{3}$ & $X_{1} X_{2}$ & $X_{2} X_{3}$ & $X_{1} X_{3}$ & $X_{1} X_{2} X_{3}$ & & & \\
\hline 1 & -1 & -1 & -1 & +1 & +1 & +1 & -1 & +1 & 7.14 & 5.43 \\
\hline 2 & +1 & -1 & -1 & -1 & +1 & -1 & +1 & +1 & 5.03 & 7.66 \\
\hline 3 & -1 & +1 & -1 & -1 & -1 & +1 & +1 & +1 & 22.61 & 25.43 \\
\hline 4 & +1 & +1 & -1 & +1 & -1 & -1 & -1 & +1 & 20.27 & 27.69 \\
\hline 5 & -1 & -1 & +1 & +1 & -1 & -1 & +1 & +1 & 10.00 & 11.69 \\
\hline 6 & +1 & -1 & +1 & -1 & -1 & +1 & -1 & +1 & 10.07 & 10.25 \\
\hline 7 & -1 & +1 & +1 & -1 & +1 & -1 & -1 & +1 & 36.23 & 32.51 \\
\hline 8 & +1 & +1 & +1 & +1 & +1 & +1 & +1 & +1 & 35.24 & 36.42 \\
\hline
\end{tabular}

+1 indicates the high level for each factor and -1 indicates the low level for each factor.

factors, Yates analysis was performed as presented in Tables 2(a) and 2(b) [12].

The regression of each polynomial equation obtained for acid degradation (see (5)) and alkali degradation (see (6)) was calculated using the following formula:

$$
R^{2}=\frac{\text { Total of significant mean square }}{\text { Total of mean square }}
$$

The regressions for acid degradation and alkali degradation polynomial equations were obtained as 0.9462 and 0.9770 , respectively, and were found to be in acceptable range.

For acid degradation, when all other mean square values were divided by the average least mean square, experimental $F$ values were found. When these were compared with tabulated $F$ values, it was found that 3360 and 648 were significantly higher than tabulated $F$ values $(98.49$ at $p<0.01$ ). Hence it had been concluded that factor $X_{2}$ (temperature) and factor $X_{3}$ (time of heating) were significant.

For alkali degradation, when all other mean square values were divided by the average least mean square, experimental $F$ values were found. When these were compared with tabulated $F$ values, it was found that 284 was significantly higher than tabulated $F$ values (98.49 at $p<0.01$ ). Hence, it was concluded that factor $X_{2}$ (temperature of heating) was significant.

To confirm the significant factors obtained for acid and alkali degradation experiments in Yates analysis and ANOVA, Pareto chart for Experiment versus Normalized squares was plotted. For acid degradation and alkali degradation, Normalized square values were plotted as depicted in Tables $3(\mathrm{a})$ and 3(b), respectively. The obtained values of effect for each experiment were ranked in decreasing order and Normalized square for each experiment was calculated using the following equation:

$$
\text { Normalized square }=100 \times \frac{E^{2}}{\sum E^{2}} .
$$

Furthermore, the representative Pareto charts were plotted as presented in Figures 3(a) and 3(b), for acid and alkali degradation, respectively. From the presented Pareto charts, it was concluded that the time of heating was the most significant factor followed by temperature in acid degradation and time of heating was the most significant factor in alkali degradation conditions.

To obtain optimized experimental conditions for acid degradation, surface response curve was generated with the help of polynomial equation obtained for acid degradation.

For acid degradation condition, by substituting value of $X_{1}=0$ (average of +1 to -1 ) in (6) and rearranging corresponding equation for $X_{3}$, the equation is obtained as

$$
X_{3}=\frac{\left(Y-18.32-10.26 X_{2}\right)}{4.56+2.59 X_{2}} .
$$

Furthermore, $Y$ values (\% degradation) were assumed to be $5 \%, 10 \%, 15 \%$, and $20 \%$; the values for $X_{3}$ at various levels of $X_{2}(-1$ to +1$)$ were calculated (Table 4$)$ and the respective surface response curve was plotted as depicted in Figure 4.

From the surface response curve for acid degradation, it was observed that, with transformed value for $X_{2}=-1$ and $X_{3}=+1,10 \%$ degradation can be expected.

Thus, optimum $10 \%$ acid degradation would result when chlorthalidone was heated using $0.55 \mathrm{M} \mathrm{HCl}$ at $55^{\circ} \mathrm{C}$ for $60 \mathrm{~min}$.

Also, for alkali degradation the actual values are obtained from transformed values using the following equation:

$$
\begin{aligned}
& \text { Transformed Value } \\
& =\frac{X-\text { the average of two levels }}{\text { one }- \text { half the difference of the levels }} .
\end{aligned}
$$

The transformed values for $X_{2}$ were obtained for $5 \%, 10 \%$, $15 \%$, and $20 \%$ of degradation, at $X_{1}=0$ and $X_{3}=0$.

$Y$ values for $5 \%$ and $20 \%$ were obtained beyond the range of -1 to +1 experimental domain, -1.32 and 0.058 , respectively. For $10 \%$ and $15 \%$ degradation, the transformed values of -0.86 and -0.44 were obtained, respectively.

The above obtained transformed values were decoded using (11). Thus, optimum $10 \%$ alkali degradation would result when chlorthalidone was heated using $0.055 \mathrm{M}$ at $56.75^{\circ} \mathrm{C}$ for $22.5 \mathrm{~min}$.

Actual experiments were performed in triplicate and subjected to chromatographic analysis. The average \% degradation of three experiments was compared with the predicted 
TABLE 2: (a) Yates analysis and ANOVA for acid degradation. (b) Yates analysis and ANOVA for alkali degradation.

(a)

\begin{tabular}{lccccccc}
\hline Expt. & $Y$ (response) & $A$ & $B$ & $C$ & Effect & Mean square & $F$ value \\
\hline$(1)(-,-,-)$ & 7 & 12 & 55 & 146 & - & - & - \\
$(2)(+,-,-)$ & 5 & 43 & 91 & -6 & -1.5 & 4.5 & 18 \\
$(3)(-,+,-)$ & 23 & 20 & -5 & 82 & 20.5 & 840.5 & 3360 \\
$(4)(+,+,-)$ & 20 & 71 & -1 & -2 & -0.5 & 0.5 & 2 \\
$(5)(-,-,+)$ & 10 & -2 & 31 & 36 & 9 & 162 & 2 \\
$(6)(+,-,+)$ & 10 & -3 & 51 & 4 & 548 \\
$(7)(-,+,+)$ & 36 & 0 & -1 & 20 & 50 & 0 \\
$(8)(+,+,+)$ & 35 & -1 & -1 & 0 & 0 & 0 & 0 \\
\hline
\end{tabular}

(b)

\begin{tabular}{lccccccc}
\hline Expt. & $Y$ (response) & $A$ & $B$ & $C$ & Effect & Mean square & $F$ value \\
\hline$(1)(-,-,-)$ & 5 & 13 & 66 & 156 & - & - & - \\
$(2)(+,-,-)$ & 8 & 53 & 90 & 8 & 2 & 2.46 \\
$(3)(-,+,-)$ & 25 & 22 & 6 & 86 & 21.5 & 924.5 & 284.46 \\
$(4)(+,+,-)$ & 28 & 68 & 2 & 6 & 1.5 & 6.5 & 72 \\
$(5)(-,-,+)$ & 12 & 3 & 40 & 24 & 6 & -1.38 \\
$(6)(+,-,+)$ & 10 & 3 & 46 & -4 & 6 & 22.2 \\
$(7)(-,+,+)$ & 32 & -2 & 0 & 6 & 1.5 & 4.5 & 1.38 \\
$(8)(+,+,+)$ & 36 & 4 & 6 & 6 & 1.5 & 4.5 & 1.38 \\
\hline
\end{tabular}

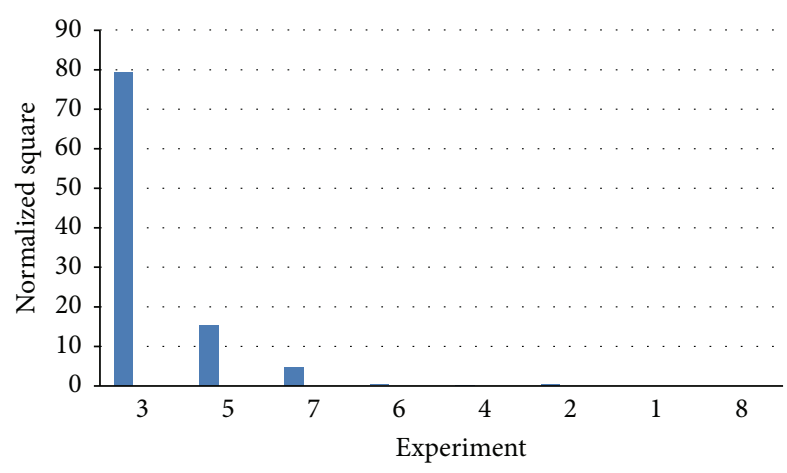

(a)

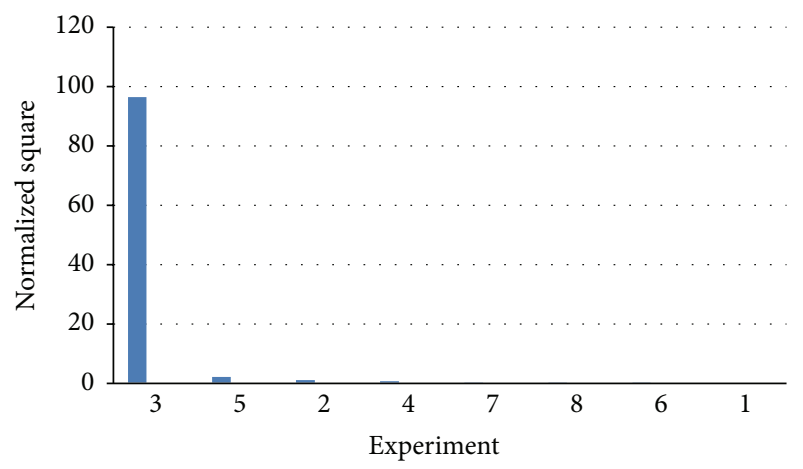

(b)

Figure 3: (a) Pareto chart for acid degradation. (b) Pareto chart for alkali degradation.

response. No significant difference was observed between predicted value and observed value.

3.4. Calibration Curve. When calibration standards in the range of $2-12 \mu \mathrm{g} / \mathrm{mL}$ were analyzed in triplicate and plot of peak area versus concentration was subjected to least square regression, the respective linear equation was

$$
y=117762 x+1167.7
$$

where $x$ is the concentration $(\mu \mathrm{g} / \mathrm{mL})$ and $y$ is the peak area $(\mu \mathrm{V})$. The correlation coefficient was 0.999 . Student's $t$ test was performed to verify the significance of experimental intercept and slope in the regression equation. According to the results, they were not significantly different from zero and one value, respectively, for $p>0.05$. The analysis of variance was applied to verify linearity of the method. From the result it has been observed that the calculated $F$ (41454.97) was greater than the tabulated $F(7.7)$ at $5 \%$ level of significance, concluding that a linear relationship exists between the peak area and concentration.

3.5. Method Validation. The results obtained for accuracy and precision studies are shown in Table 5 . The \% recovery close to $100 \%$ and the low values of $\%$ RSD suggest an acceptable accuracy of the method. Furthermore, the intraday and interday results at each level were subjected to one-way analysis of variance and $F$ values for each level were determined as the ratio of between mean square (BMS) to within mean square (WMS):

$$
\left(F=\frac{\mathrm{BMS}}{\mathrm{WMS}}\right)
$$

see $[13]$. 
TABLE 3: (a) Normalized square values for acid degradation. (b) Normalized square values for alkali degradation.

(a)

\begin{tabular}{lcccc}
\hline Rank & Expt. number & Value $(E)$ & $E^{2}$ & Normalized square \\
\hline 1 & $(3)(-,+,-)$ & 20.5 & 420.25 & 79.32 \\
2 & $(5)(-,-,+)$ & 9 & 81 & 15.29 \\
3 & $(7)(-,+,+)$ & 5 & 25 & 4.71 \\
4 & $(6)(+,-,+)$ & 1 & 1 & 0.19 \\
5 & $(8)(+,+,+)$ & 0 & 0 & 0 \\
6 & $(4)(+,-,+)$ & -0.5 & 0.25 & 0.57 \\
7 & $(2)(+,-,-)$ & -1.5 & 2.25 & 0.42 \\
8 & $(8)(-,-,-)$ & - & - & - \\
\hline
\end{tabular}

(b)

\begin{tabular}{lcccc}
\hline Rank & Expt. number & Value $(E)$ & $E^{2}$ & Normalized square \\
\hline 1 & $(3)(-,+,-)$ & 21.5 & 462.25 & 96.52 \\
2 & $(5)(-,-,+)$ & 3 & 9 & 1.87 \\
3 & $(2)(+,-,-)$ & 2 & 4 & 0.84 \\
4 & $(4)(+,+,-)$ & 1.5 & 2.25 & 0.47 \\
5 & $(7)(-,+,+)$ & 0.75 & 0.5625 & 0.12 \\
6 & $(8)(+,+,+)$ & 0.75 & 0.5625 & 0.12 \\
7 & $(6)(+,-,+)$ & -0.5 & 0.25 & 0.05 \\
8 & $(1)(-,-,-)$ & - & - & - \\
\hline
\end{tabular}

TABLE 4: Transformed values $\left(X_{3}\right)$ at various levels of $X_{2}$ for acid degradation conditions.

\begin{tabular}{lcccc}
\hline$X_{3}\left(\right.$ for $\left.X_{1}=0\right)$ & $5 \%$ & $10 \%$ & $15 \%$ & $20 \%$ \\
\hline-1 & -1.55 & 0.98 & 3.52 & 6.06 \\
-0.5 & -2.51 & -0.98 & 0.55 & 2.09 \\
0 & -2.92 & -1.82 & -0.73 & 0.37 \\
0.5 & -3.15 & -2.30 & -1.44 & -0.59 \\
1 & -3.30 & -2.60 & -1.90 & -1.2 \\
\hline
\end{tabular}

The obtained $F$ values were found to be less than the tabulated $F_{(2,6)}$ at $\alpha=0.05$ (tabulated $F$ value $=5.14$ ). These indicated that there was no significant difference between intraday variability and interday variability, suggesting good intermediate precision of the method. A plot of quantity added to the quantity obtained resulted in a straight line with the slope of 1.1667 and the intercept of 0.998 , encompassing 1 and 0 , respectively. This indicated the linearity of the method in the selected range of $80-120 \%$ of the label claimed. Based on the SD of the response and the slope, the limit of detection (DL) was found to be $0.678 \mu \mathrm{g} / \mathrm{mL}$ and limit of quantitation (QL) was $1.872 \mu \mathrm{g} / \mathrm{mL}$. The chromatograms of blank and placebo solutions showed no interfering peak at the retention time of the drug indicating specificity of the developed method.

3.6. Analysis of Formulation. The drug content was found to be $101.28 \pm 1.17 \%$ with a $\%$ RSD of 1.16 . The $\%$ RSD value

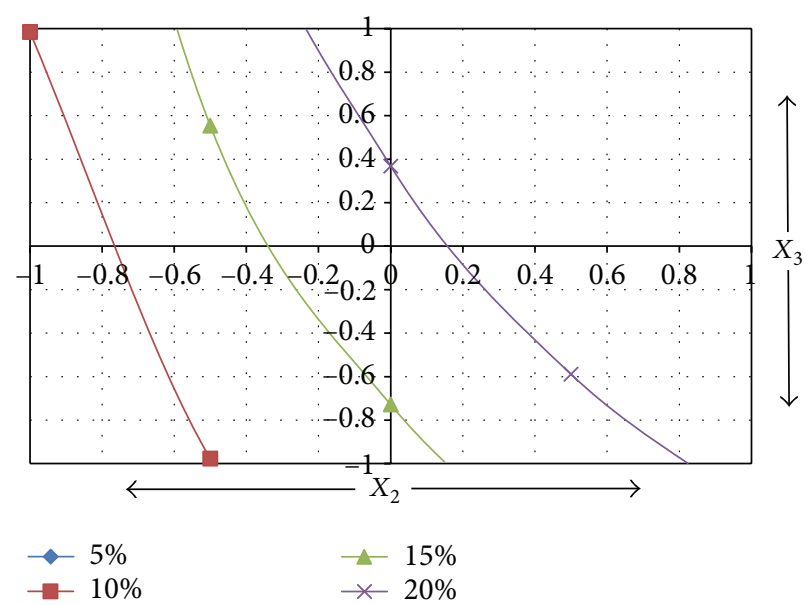

FIGURE 4: Surface response curve of transformed values for acid degradation from -1 to +1 range.

indicated the suitability of the method for routine analysis of chlorthalidone in formulation.

\section{Conclusion}

The developed HPLC technique is precise, specific, accurate, and stability-indicating. Validation of the method proved that the method is suitable for the analysis of chlorthalidone in tablet formulation without any interference from common excipients or potential degradation product of chlorthalidone 
TABLE 5: Accuracy and precision studies.

\begin{tabular}{|c|c|c|c|c|c|c|}
\hline \multirow{2}{*}{ Amount added (mg) } & \multicolumn{3}{|c|}{ Amount found (mg) } & \multirow{2}{*}{ Within mean square } & \multirow{2}{*}{ Between mean square } & \multirow{2}{*}{$F$ value } \\
\hline & Day 1 & Day 2 & Day 3 & & & \\
\hline \multirow{3}{*}{$80 \%(10 \mathrm{mg})$} & 10 & 10.02 & 10 & \multirow{6}{*}{0.0009} & \multirow{6}{*}{0.00028} & \multirow{6}{*}{0.3086} \\
\hline & 10.05 & 10.03 & 10.09 & & & \\
\hline & 10.03 & 10.03 & 10.04 & & & \\
\hline Mean & 10.03 & 10.03 & 10.04 & & & \\
\hline SD & 0.252 & 0.058 & 0.451 & & & \\
\hline$\%$ RSD & 0.251 & 0.058 & 0.449 & & & \\
\hline \multirow{3}{*}{$100 \%(12.5 \mathrm{mg})$} & 12.45 & 12.42 & 12.45 & \multirow{6}{*}{0.00208} & \multirow{6}{*}{0.00174} & \multirow{6}{*}{0.8396} \\
\hline & 12.42 & 12.41 & 12.42 & & & \\
\hline & 12.33 & 12.49 & 12.46 & & & \\
\hline Mean & 12.4 & 12.44 & 12.44 & & & \\
\hline SD & 0.62 & 0.44 & 0.208 & & & \\
\hline$\%$ RSD & 0.503 & 0.350 & 0.167 & & & \\
\hline \multirow{3}{*}{$120 \%$ (15 mg) } & 14.09 & 14.01 & 14.032 & \multirow{6}{*}{0.00078} & \multirow{6}{*}{0.0012} & \multirow{6}{*}{1.508} \\
\hline & 14.02 & 14.03 & 14.07 & & & \\
\hline & 14.06 & 14.04 & 14.09 & & & \\
\hline Mean & 14.06 & 14.03 & 14.06 & & & \\
\hline SD & 0.351 & 0.152 & 0.295 & & & \\
\hline$\%$ RSD & 0.251 & 0.110 & 0.209 & & & \\
\hline
\end{tabular}

and excipients. The developed method can be used for routine analysis of chlorthalidone tablets or for assay of chlorthalidone tablets from stability batches.

\section{Competing Interests}

The authors declare that they have no competing interests.

\section{Acknowledgments}

The authors are thankful to the management and trustees of Mumbai Educational Trust's Bhujbal Knowledge City, Nashik, for providing necessary chemicals and analytical facilities and to Trichem Laboratories Ltd., Mumbai, for providing pharmaceutical grade chlorthalidone as gift sample.

\section{References}

[1] M. Bakshi and S. Singh, "Development of validated stabilityindicating assay methods-critical review," Journal of Pharmaceutical and Biomedical Analysis, vol. 28, no. 6, pp. 1011-1040, 2002.

[2] S. Singh and M. Bakshi, Guidance on Conduct of Stress Testing to Determine Inherent Stability of Drugs, Pharmaceutical Technology, 2000.

[3] S. Sonawane and P. Gide, "Application of experimental design for the optimization of forced degradation and development of a validated stability-indicating LC method for luliconazole in bulk and cream formulation," Arabian Journal of Chemistry, 2012.

[4] M. Kurmi, S. Kumar, B. Singh, and S. Singh, "Implementation of design of experiments for optimization of forced degradation conditions and development of a stability-indicating method for furosemide," Journal of Pharmaceutical and Biomedical Analysis, vol. 96, pp. 135-143, 2014.

[5] J. M. O'Neil, The Merck Index: An Encyclopedia of Chemicals, Drugs, and Biologicals, Merck \& Co, Whitehouse Station, NJ, USA, 14th edition, 2006.

[6] R. A. Mhaske, D. J. Garole, A. A. Mhaske, and S. Sahasrabudhe, "RP-HPLC method for simultataneous determination of amlodipine Besylate, Valsartan, Telmisartan, Hydrochlorothiazide and Chlorthalidone: application to commercially available drug products," International Journal of Pharmaceutical Sciences, vol. 3, no. 1, pp. 141-149, 2012.

[7] M. S. Charde, A. S. Welankiwar, and R. D. Chakole, "Development of force degradation profile of atenolol and Chlorthalidone in combine tablet dosage form by (RP-HPLC)," International Journal of Pharmaceutical Chemistry, vol. 4, no. 3, pp. 9298, 2014.

[8] G. S. Kumar, V. Ramya, S. Mondal, and S. P. Kumar, "Development and validation of RP-HPLC method for simultaneous estimation of atenolol and chlorthalidone from pharmaceutical formulation," International Research Journal of Pharmacy, vol. 3, no. 10, pp. 215-219, 2012.

[9] ICH Harmonised Tripartite Guideline, "Stability testing of new drug substances and products Q1A(R2)," in International Conference on Harmonization of Technical Requirements for Registration of Pharmaceuticals for Human Use, February 2003.

[10] ICH Harmonized Triplicate Guidelines, "Validation of analytical procedures: text and methodology, Q2R1," in International Conference on Harmonization of Technical Requirements for Registration of Pharmaceuticals for Human Use, 2005.

[11] L. R. Synder, J. J. Kirkland, and J. L. Giajch, Practical HPLC Method Development, A Wiley Inter Science Publication, 2nd edition, 1997.

[12] S. Sonawane and P. Gide, "An experimental design approach for the forced degradation studies and development of a stabilityindicating lc method for eplerenone in tablets," Journal of Liquid 
Chromatography and Related Technologies, vol. 34, no. 17, pp. 2020-2031, 2011.

[13] S. Bolton and C. Bon, Pharmaceutical Statistics Practical and Clinical Applications, vol. 135 of Drugs and the Pharmaceutical Sciences, Marcel Dekker, New York, NY, USA, 4th edition, 2004. 

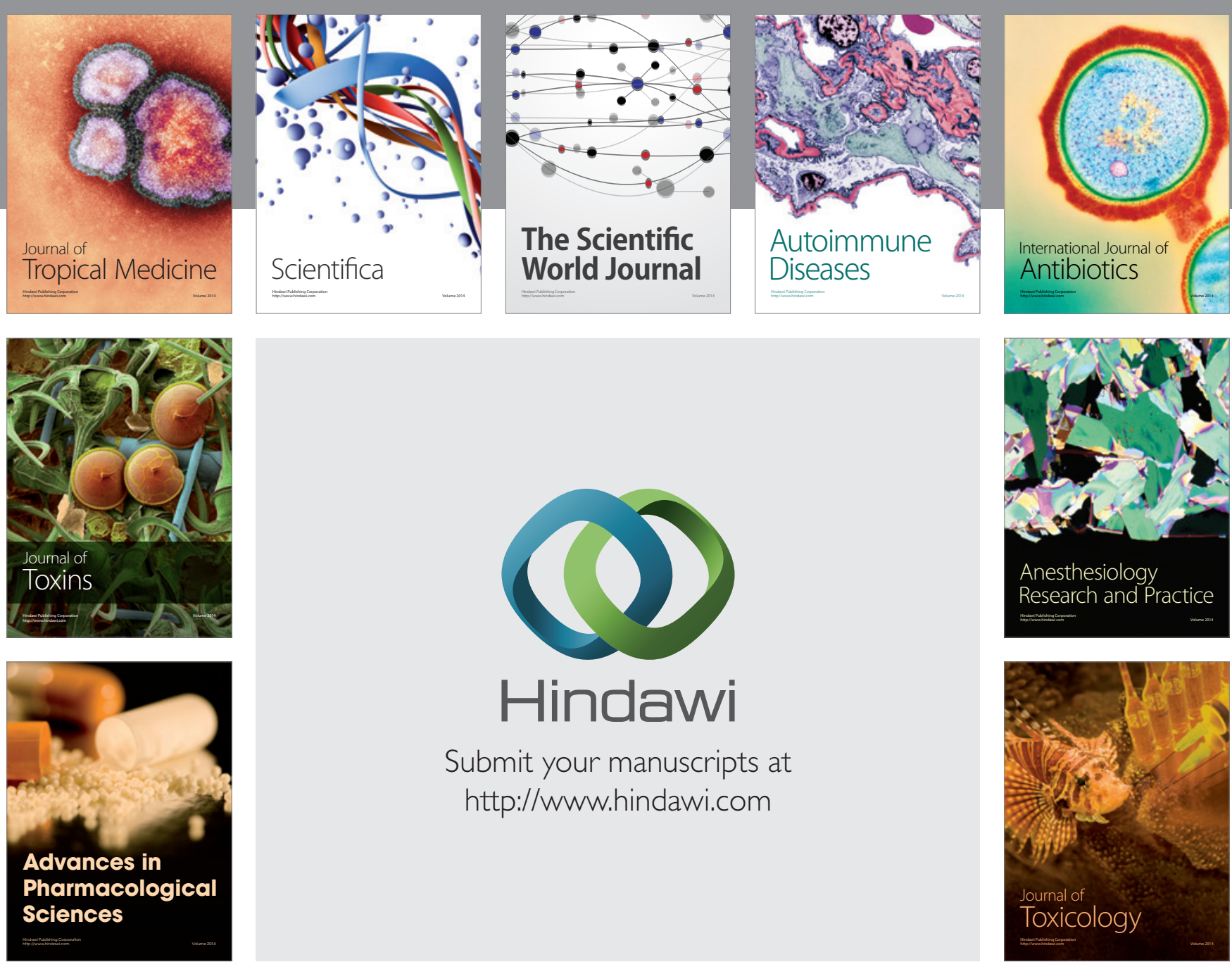

\section{Hindawi}

Submit your manuscripts at

http://www.hindawi.com
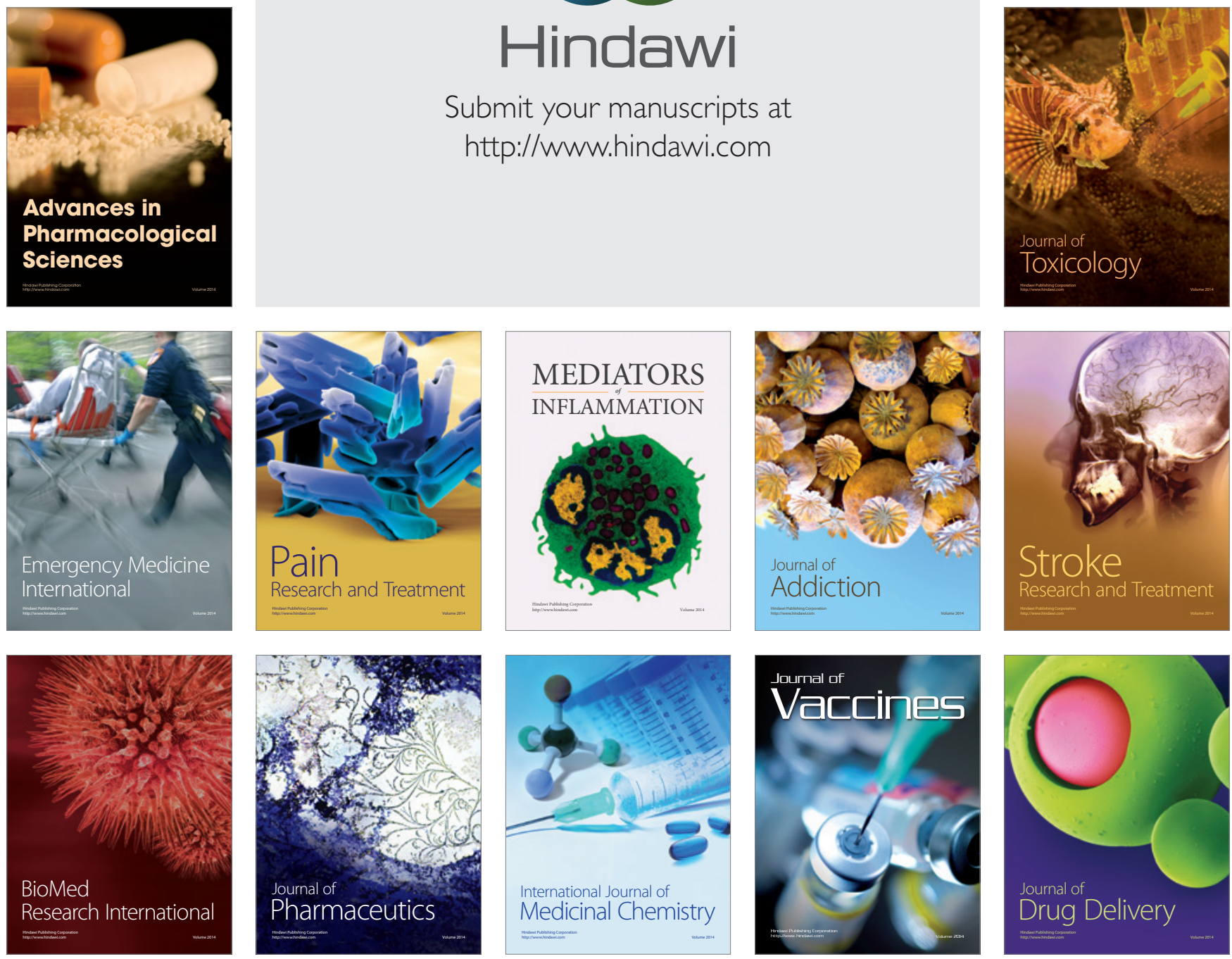